\title{
Electroretinogram and Visual Evoked Potentials in Patients with Type II Diabetes Mellitus
}

\author{
Asmaa Saadoun*, Mahmoud Rajab, Habib Yousef \\ Ophthalmology Department, Tishreen Hospital, Faculty of Medicine, Tishreen University, Lattakia, Syria \\ Email address: \\ asmaa.saadoun@tishreen.edu.sy (A. Saadoun), mahmoud rajab@tishreen.edu.sy (M. Rajab), habib.yousef @tishreen.edu.sy (H. Yousef) \\ ${ }^{*}$ Corresponding author
}

\section{To cite this article:}

Asmaa Saadoun, Mahmoud Rajab, Habib Yousef. Electroretinogram and Visual Evoked Potentials in Patients with Type 11 Diabetes Mellitus. American Journal of Biomedical and Life Sciences. Vol. 8, No. 6, 2020, pp. 212-219. doi: 10.11648/j.ajbls.20200806.14

Received: July 7, 2020; Accepted: July 22, 2020; Published: December 4, 2020

\begin{abstract}
Background: Retinopathy is a severe and common complication of diabetes. The pathology seems to be characterized not only by the involvement of retinal micro vessels but also by a real neuropathy. Before the onset of micro vascular lesions, the retina of the eye undergoes subtle functional changes that are not detectable by fundus photography. Electrophysiological investigations allow a more detailed study of the visual function. These techniques are safe, repeatable, quick, and objective. Objective: To study pattern electroretinogram (PERG) and pattern reversal visual evoked potentials (PRVEP) in type 2 diabetic patients without diabetic retinopathy (DR) or with mild non-proliferative DR (mNPDR) to detect changes by comparing with those of healthy control. And to assess the correlation of the parameters with diabetes duration and the level of Glycosylated Haemoglobin A1c (HbAlc). Materials and Methods: It was a cross-sectional study, included two groups (diabetic patients and the healthy). Age range was preset at 40-65 years. For all the participants, a detailed clinical history was collected, a comprehensive ophthalmic examination and thorough blood investigations were performed, then \{PRVEP (60', 15'), PERG\} were recorded and (waveform, peak time, amplitude) of tests components were analyzed. Results: Mean (P100, N135) peak times of PRVEP were statistically significantly delayed in (50) eyes of type 2 diabetics without DR when compared to (36) eyes of control ( $\mathrm{p}$-value $<0.01$ ), abnormalities in waveforms like (double peaks, broad peak) were also observed in diabetics. There were alterations in other parameters (amplitudes of PRVEP, peak times and amplitudes of PERG) but the changes were not statistically significant. No statistically significant changes were found in (6) eyes of diabetic patients with mNPDR. No statistically significant correlation was obtained between diabetes duration or the level of HbAlc and delay of peak times or reduce amplitudes in patients. Conclusions: Electrophysiological tests are sensitive and useful investigations for the early identification of visual dysfunctions before the development of overt retinopathy in type 2 diabetics. PRVEP is more sensitive than PERG to monitor alterations and it may be sufficient to screen the patients in this stage.
\end{abstract}

Keywords: Diabetic Retinopathy, Pattern Reversal Visual Evoked Potentials, Pattern Electroretinogram, Diabetes Duration, Glycosylated Haemoglobin A1c

\section{Introduction}

Diabetes mellitus (DM) is recognized as the global epidemic, presently one of the most important challenges in healthcare [1]. Chronic hyperglycemia of diabetes is associated with long term damage, dysfunction and the failure of various organs, especially the eyes, kidneys, nerves, the heart and the blood vessels [2]. An estimated 463.0 million adults aged 20-79 years worldwide (9.3\% of all adults in this age group) have diabetes. Based on the 2019 estimates, by 2030 a projected 578.4 million, and by $2045,700.2$ million adults, will be living with diabetes. Type 2 diabetes is the most common type of diabetes, accounting for around $90 \%$ of all diabetes worldwide [3]. Diabetic retinopathy (DR) is a serious and common complication of diabetes [4], and remains the leading cause of blindness among working aged adults around the world [5]. DR can be of two types, non-proliferative and proliferative, the advanced stage is proliferative diabetic retinopathy. Visual acuity is significantly reduced as DR progresses, but this sensitivity is insufficient to identify the early stages of DR [4]. 
The diagnosis and treatment of DR are focused on vascular abnormalities (such as microaneurysms, exudates, intraretinal haemorrhages, edema) which are usually detected by ophthalmoscopic, angiofluorographic [6] or optical coherence tomography (OCT) [6, 7]. A patient diagnosed with DR has approximately a 50\% chance of losing vision in 5 years, which in itself calls for efforts to detect retinopathy at the earliest possible stage [8].

DR is classically considered a microvascular disease [9]. However, growing evidences suggest that retinal diabetic neuropathy (RDN) also makes an important contribution to the disease and this process begins soon after the onset of diabetes, in the absence of gross vascular lesions; this neuropathy is observed structurally as neural apoptosis, ganglion cell (GC) loss, reactive gliosis, thinning of the inner retina $[10,11]$, changes in neurotransmitter and synapse function [10]; and functionally, as deficits in the dark adaptation, contrast sensitivity, color vision, and microperimetric and perimetric psychophysical testing [11]. Over the past two decades, the advent of new neurophysiological techniques to assess retinal and brain (optic tract) function, from the retinal pigment epithelium (RPE) to the visual cortex, such as electroretinography (ERG) and the measurement of visual evoked potentials (VEP), allowed a more detailed study of the visual function. These techniques are safe, repeatable, quick, and objective [7, 12].

The aim of this study is to detect the role of electrophysiological investigations in determining the early functional changes of visual pathways in type 2 diabetic patients who had either normal fundi or microaneurysms only, by comparing (PERG, PRVEP) parameters between them and the healthy participants. And assessing the correlation of the responses with diabetes duration and the level of $\mathrm{HbAlc}$.

\section{Materials and Methods}

\subsection{Design}

A cross sectional study.

\subsection{Subjects}

Two groups (The diabetic group and the healthy one) were recruited from the outpatient ophthalmic clinic of Tishreen University Hospital in Lattakia, Syria. From December 2018 till January 2020.

\subsubsection{Ethical Considerations}

An informed consent and ethical committee clearance were taken for this study.

\subsubsection{Inclusion Criteria}

Patients with known history of type 2 diabetes mellitus or recently diagnosed, either on treatment or discontinued treatment, without DR or with mild NPDR (microaneurysms only), age group: 40 to 65 years / both genders. Age and sexmatched subjects who were free of (ocular diseases, diabetes, hypertension, or other systemic diseases) were recruited as control.

\subsubsection{Exclusion Criteria}

Best-corrected visual acuity (BCVA) worse than 20/20, high spherical or cylindrical $\geq \pm 3$ dioptric refractive errors, any type of the previous retinal treatments (laser photocoagulation, intravitreal drugs), hazy media, other ocular diseases such as (cataract, uveitis or macular degeneration, glaucoma, etc.), intraocular pressure (IOP) $>21$ $\mathrm{mm} \mathrm{Hg}$, having a history of systemic diseases like (neurological, endocrinological and vascular except for hypertension which has caused no changes through fundus appearance and ECG as well as clinical examination yet), fundoscopic changes except microaneurysms or peripheral retinal degenerations, OCT abnormalities, drugs acting on central nervous system, habitual history of alcohol drinking, any uncooperative or febrile patient.

\subsection{Procedures}

Personal details of all the participants such as (name, age, sex, phone number, and detailed clinical history including the duration of diabetes and glycemic control) were written in a questionnaire.

All subjects underwent a comprehensive ophthalmological examination: refraction, BCVA, IOP measured by the Goldmann applanation tonometer, anterior segment examination, dilatation and fundoscopy (slit lamp with $+90 \mathrm{~d}$ lens, indirect ophthalmoscopy), and OCT. Thorough blood investigations (fasting plasma glucose, blood urea nitrogen, creatinine, cholesterol, triglycerides, C-reactive protein, complete blood count, HbAlc). oxygen saturation, arterial blood pressure and pulse rate were recorded.

Retimax (CSO, Italy) was used for recording PRVEP and PERG in accordance to the International Society for Clinical Electrophysiology of Vision (ISCEV) guidelines [13, 14]. For the best tests results, subjects were advised to come without applying any oil or hair chemicals to their scalps, were instructed to have an adequate sleep the previous night to prevent the effect of drowsiness on the responses. On the day of procedure, subjects were advised to seat comfortably at a distance of $100 \mathrm{~cm}$ (1metre) from monitor screen and explained for about the test to ensure full cooperation, preparation of scalp skin was done before electrode application. For recording PRVEP, surface electrodes were placed with electrode paste and gel according to the international 10/20 system of electrode placement, the active electrode (silver cup skin) was placed on the scalp over the visual cortex at $\mathrm{Oz}$, the reference electrode on $\mathrm{Fz}$ and the ground electrode on Fpz [13, 15] "figure 1". For PERG, H-K loop electrodes that contact the bulbar conjunctiva were used, the reference electrodes (silver cup skin) were placed over both temples, approximately $1 \mathrm{~cm}$ from the ipsilateral orbital rim, and the ground electrode was placed on the $\mathrm{Fz}[14,15]$ "figure 1". The inter-electrode impedance was checked and maintained below $5 \mathrm{KOhm}$ in all recordings. 


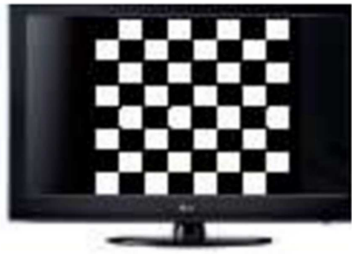

a

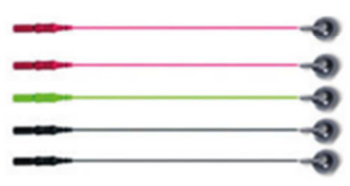

$\mathrm{c}$

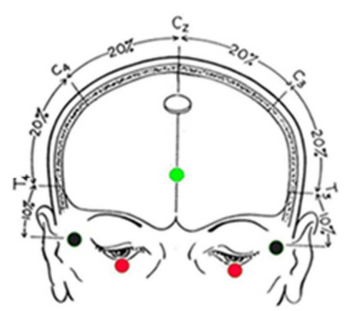

$\mathrm{e}$

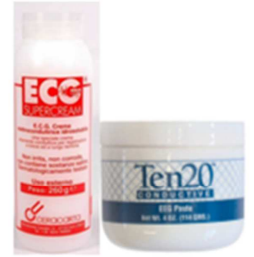

$\mathrm{b}$

d

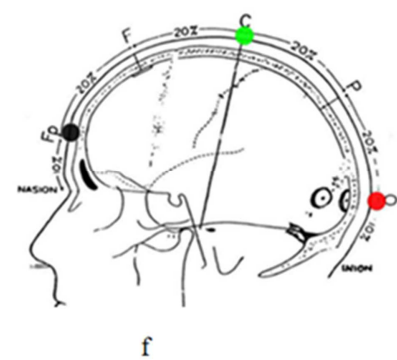

Figure 1. Retimax equipments in the ophthalmological clinic \{(a) Pattern stimulator, (b) gel and adhesive paste, (c) silver cup skin electrodes, (d) H-K loop electrodes \}, and the specific electrode contact points for tests \{(e) for ERG, (f) for VEP\} [15].

All the tests were performed with the patients wearing the best refractive correction for viewing distance. Subjects were being instructed to fix their gaze at the center of the checker board, a red cross, to avoid interference in potentials due to the movement of the eyeball. The room was made quiet and with uniform light levels. Monocular stimulation was applied for VEP, the other eye was covered with opaque material, and binocular stimulation for PERG. Automatic artefact rejection was used. The type of stimuli presented was pattern stimuli with a black and white checker-board reversing alternately at the rate of $2 \mathrm{~Hz}$, contrast $99 \%$ and a full-field display. The check size was (17 and 68) min of arc for PRVEP (will be symbolized as: PRVEP15' and PRVEP60' respectively) and (25.50) min for PERG. Responses to 100 stimuli were recorded. The signals were amplified, displayed as a waveform. The amplifier band-pass filters were set at 1-30 Hz. To test trial-to-trial variability, all tests were repeated in the same session after a break of (3-5) min.

PRVEP and PERG consist of a series of components of opposite polarity, negative ones (denoted as $\mathrm{N}$ ) and positive (denoted as P).

The usual PRVEP waveform is the initial negative peak (N75), followed by a large positive peak (P100) and followed by another negative peak (N135) [13]. The usual PERG waveform is the initial negative peak (N35), followed by a positive peak (P50) and followed by another negative peak (N95) [14].

For the evaluation of PRVEP and PERG alterations \{waveform, peak time (or latency for VEP) (in millisecond) and amplitude (in microvolt) $\}$ of components were analyzed. Mean values of parameters were compared between the two groups (patients and control).

\subsection{Statistical Analysis}

Descriptive statistical: the quantitative data (central tendency, Measures of dispersion), and the qualitative ones (Frequencies and Percentile Values). Inferential Statistical: chi-square test, one-way analysis of variances (ANOVA), independent t-test, correlation analyses were performed using Pearson's correlation coefficient for parametric data. The data were analyzed by SPSS (Statistical Package for the Social Sciences) version 19. The statistical analysis was done at significance level of $5 \%$.

\section{Results}

A total of 56 eyes from 32 diabetic patients and 36 eyes from 18 healthy subjects met our criteria and were included in this study. Subjects with DM were subdivided into two groups according to the presence or absence of DR, the first group consisted of 50 eyes without DR and the second group consisted of 6 eyes with mNPDR. The median age of the participants was 49 years. The characteristics of the three groups are presented in Table 1. There were no statistical differences in age and gender between control and diabetic groups $(\mathrm{p} \geq 0.05)$, and no statistical differences in diabetes duration, glycemic status (fasting plasma glucose, HbA1c) between diabetic groups.

Table 1. Demographic profile of the subjects in the study groups.

\begin{tabular}{llll}
\hline Study subjects & Control & Without DR & With mNPDR \\
\hline Sex (M/F) & $16 / 20$ & $25 / 25$ & $5 / 1$ \\
age (year) & $47.8 \pm 4.9$ & $50.2 \pm 5.7$ & $48.1 \pm 3.8$ \\
Diabetes duration (year) & - & $4.1 \pm 3.4$ & $6.3 \pm 4.1$ \\
FPG (mg/dl) & - & $155.7 \pm 46.6$ & $147.1 \pm 36.4$ \\
HbAlc (\%) & - & $7.09 \pm 1.5$ & $7.5 \pm 2.1$ \\
\hline
\end{tabular}

FPG: Fasting Plasma Glucose, HbA1c: Glycosylated haemoglobin A1c.

We have observed waveform alterations in both groups, and they were most common in diabetics such as: wavy arm in 12 eyes, bifid P100 in 9 eyes, negative wave symbols shift from their lowest point in most patients' eyes. And other alterations only in patients such as M shaped peak in 2eyes, and broad peak in 2eyes "Figure 2".

The mean \pm standard deviation of peak times and amplitudes for PRVEP and PERG of the three groups are given in Tables (2 and 3 ). There were statistically significant differences between control and patients without DR in mean 
P100 peak times of PRVEP $\left(60^{\prime}\right.$ and $\left.15^{\prime}\right)(p=0.0001,0.0001)$ respectively and N135 peak times of PRVEP (60' and 15') $(\mathrm{p}=0.002,0.0001)$ respectively (Table 2$)$.

The range of P100 peak time in PRVEP60' in the three groups was as follow:(control: 91.41-107.81, without DR: 97.85-117.77, with mNPDR: 95.51-100.78) "Figure 3", and The range of P100 peak time in PRVEP15' was as follow (control: 93.75-108.98, without DR: 95.51-113.67, with mNPDR: 96.6-106.64) "Figure 4". Gender influence was found as increased mean P100 peak times in healthy and diabetic males without DR, but with no statistical significance.

There were no significant differences between control and patients groups in (N35, P50, N95) peak times and amplitudes (Table 3).

There was no statistically significant positive correlation between diabetes duration or the level of $\mathrm{HbAlc}$ and peak times of PRVEP (60' and 15') in diabetic patients. Also, there was no statistically significant negative correlation between diabetes duration or the level of HbA1c and amplitudes of PRVEP in diabetic patients except (N75-P100) amplitude of PRVEP15' (Table 4).

No significant association was obtained between duration of diabetes mellitus or HbA1c level and PERG parameters (Table 5).

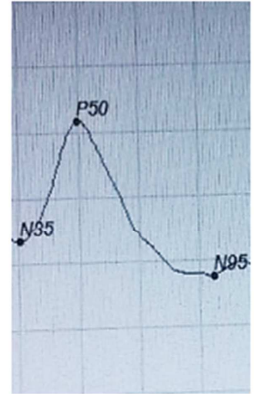

a

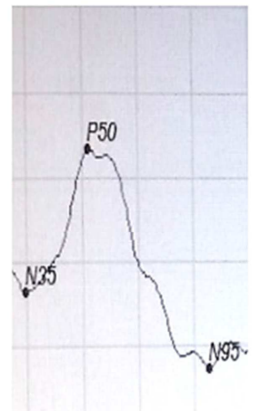

d

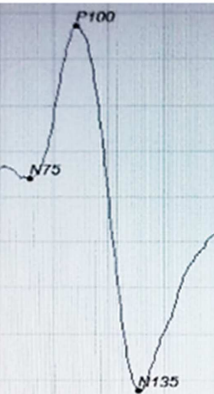

$\mathrm{b}$

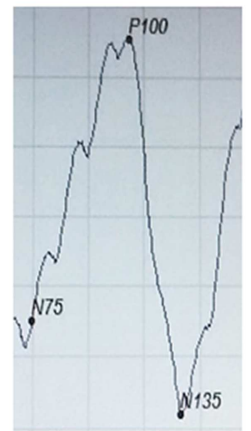

e

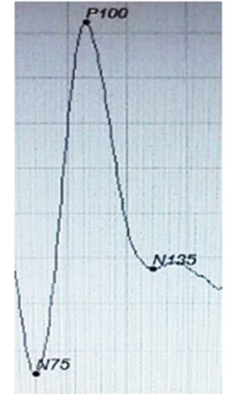

c

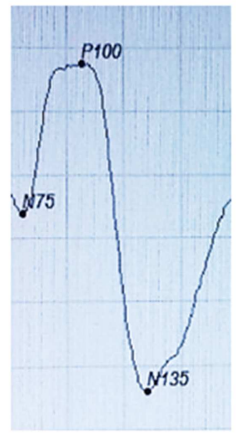

f
Figure 2. Examples of healthy waveforms \{(a) PERG, (b) PRVEP60', (c) PRVEP15' $\}$ and diabetics alterations $\{(d)$ wavy arm in PERG,(e) $M$ shaped peak in PRVEP60', (f) Broad peak in PRVEP60'\}.

Table 2. Comparison of mean PRVEP parameters between DM type2 groups and control.

\begin{tabular}{|c|c|c|c|c|}
\hline PRVEP parameters & Control (36 eyes) & Without DR (50 eyes) & With mNPDR (6 eyes) & P-value \\
\hline $60^{\prime} \mathrm{N} 75 \mathrm{~ms}$ & $66.7 \pm 5.6$ & $65.5 \pm 6.3$ & $63.5 \pm 4.2$ & 0.4 \\
\hline $15^{\prime} \mathrm{N} 75 \mathrm{~ms}$ & $74.5 \pm 5.8$ & $77.1 \pm 7.2$ & $75.6 \pm 4.2$ & 0.2 \\
\hline $60^{\prime} \mathrm{P} 100 \mathrm{~ms}$ & $99.8 \pm 4.3$ & $104.9 \pm 5.1$ & $99.2 \pm 2.4$ & 0.0001 \\
\hline $15^{\prime} \mathrm{P} 100 \mathrm{~ms}$ & $101.1 \pm 3.4$ & $105.9 \pm 4.1$ & $101.5 \pm 3.6$ & 0.0001 \\
\hline $60^{\prime} \mathrm{N} 135 \mathrm{~ms}$ & $138.2 \pm 10.1$ & $143.2 \pm 6.6$ & $140.8 \pm 7.1$ & 0.002 \\
\hline $15^{\prime} \mathrm{N} 135 \mathrm{~ms}$ & $133.2 \pm 7.6$ & $140.8 \pm 6.2$ & $138.3 \pm 5.8$ & 0.0001 \\
\hline $60^{\prime} \mathrm{N} 75 \mu \mathrm{V}$ & $2.08 \pm 1.5$ & $1.8 \pm 1.3$ & $1.4 \pm 0.8$ & 0.4 \\
\hline 60' N75-P100 $\mu \mathrm{V}$ & $12.06 \pm 3.6$ & $11.6 \pm 3.2$ & $10.9 \pm 3.2$ & 0.7 \\
\hline $15^{\prime} \mathrm{N} 75-\mathrm{P} 100 \mu \mathrm{V}$ & $14.7 \pm 4.9$ & $13.7 \pm 4.2$ & $10.5 \pm 2.1$ & 0.1 \\
\hline $60^{\prime} \mathrm{P} 100-\mathrm{N} 135 \mu \mathrm{V}$ & $15.8 \pm 6.7$ & $15.8 \pm 6.1$ & $14.9 \pm 7.3$ & 0.9 \\
\hline $15^{\prime} \mathrm{P} 100-\mathrm{N} 135 \mu \mathrm{V}$ & $17.8 \pm 4.4$ & $18.4 \pm 6.3$ & $15.4 \pm 6.2$ & 0.4 \\
\hline
\end{tabular}

ms: millisecond, $\mu \mathrm{V}$ : microvolt.

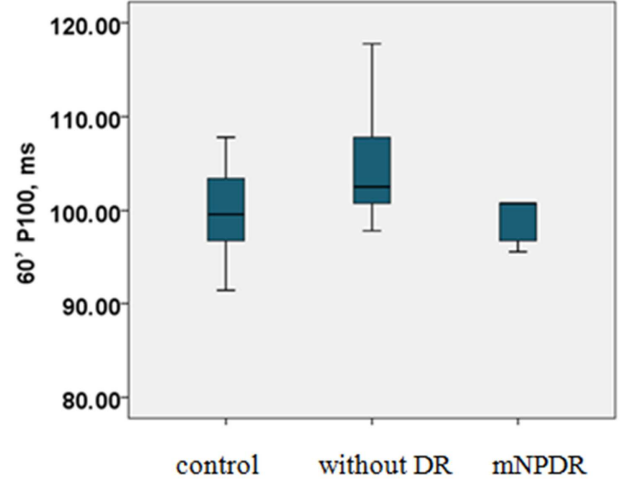

Figure 3. Distribution of P100 peak time values of PRVEP60' in the study groups.

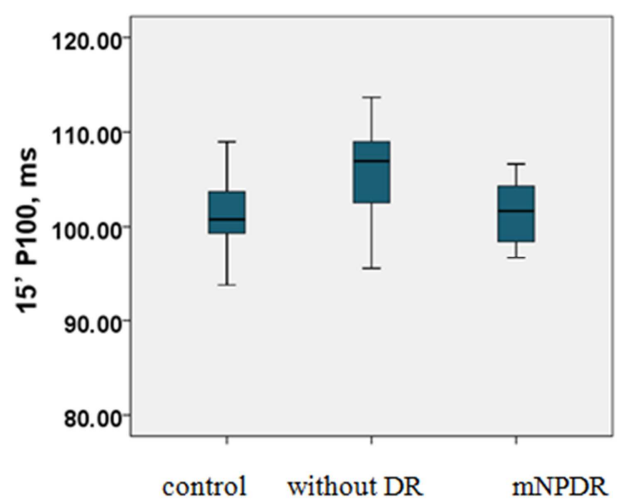

Figure 4. Distribution of $P 100$ peak time values of PRVEP15' in the study groups. 
Table 3. Comparison of mean PERG parameters between DM type2 groups and control.

\begin{tabular}{lllll}
\hline PERG parameters & Control (36 eyes) & Without DR (50 eyes) & With mNPDR (6 eyes) & P-value \\
\hline N35 ms & $26.4 \pm 2.1$ & $26.3 \pm 4.1$ & $26.4 \pm 2.1$ & 0.8 \\
P50 ms & $51.9 \pm 2.1$ & $51.7 \pm 4.8$ & $51.9 \pm 2.1$ & 0.9 \\
N95 ms & $100.8 \pm 7.3$ & $103.8 \pm 8.6$ & $100.8 \pm 7.3$ & 0.2 \\
N35 $\mu \mathrm{V}$ & $1 \pm 0.8$ & $0.97 \pm 0.8$ & $0.92 \pm 0.3$ & 0.9 \\
N35-P50 $\mu \mathrm{V}$ & $6.5 \pm 1.4$ & $5.7 \pm 2.1$ & $5.8 \pm 1.6$ & 0.1 \\
P50-N95 $\mu \mathrm{V}$ & $9.2 \pm 1.9$ & $8.2 \pm 2.4$ & $8.4 \pm 2.5$ & 0.1 \\
PERG Ratio (N95: P50) & $1.43 \pm 0.1$ & $1.48 \pm 0.2$ & $1.47 \pm 0.1$ & 0.5 \\
\hline
\end{tabular}

ms: millisecond, $\mu \mathrm{V}$ : microvolt.

Table 4. Correlation of PRVEP parameters with diabetes duration and the HbAlc level.

\begin{tabular}{|c|c|c|c|c|}
\hline \multirow{2}{*}{ PRVEP parameters } & \multicolumn{2}{|c|}{ Correlation with diabetes duration } & \multicolumn{2}{|c|}{ Correlation with the HbA1c level } \\
\hline & $\mathbf{r}$ & P-value & $\mathbf{r}$ & P-value \\
\hline $60^{\prime} \mathrm{N} 75 \mathrm{~ms}$ & 0.009 & 0.9 & 0.2 & 0.1 \\
\hline $15^{\prime} \mathrm{N} 75 \mathrm{~ms}$ & 0.08 & 0.5 & -0.1 & 0.2 \\
\hline 60' P100 ms & -0.28 & 0.03 & -0.1 & 0.3 \\
\hline 15' P100 ms & -0.1 & 0.3 & -0.1 & 0.2 \\
\hline $60^{\prime} \mathrm{N} 135 \mathrm{~ms}$ & 0.09 & 0.4 & 0.03 & 0.8 \\
\hline $15^{\prime} \mathrm{N} 135 \mathrm{~ms}$ & -0.1 & 0.2 & 0.09 & 0.5 \\
\hline $60^{\prime} \mathrm{N} 75 \mu \mathrm{V}$ & 0.3 & 0.02 & -0.2 & 0.1 \\
\hline $15^{\prime} \mathrm{N} 75 \mu \mathrm{V}$ & 0.04 & 0.7 & -0.2 & 0.07 \\
\hline 60'N75-P100 $\mu \mathrm{V}$ & 0.05 & 0.6 & 0.08 & 0.5 \\
\hline 15'N75-P100 $\mu \mathrm{V}$ & 0.1 & 0.2 & -0.3 & 0.03 \\
\hline 60' P100-N135 $\mu \mathrm{V}$ & -0.08 & 0.5 & -0.01 & 0.9 \\
\hline 15' P100-N135 $\mu \mathrm{V}$ & -0.06 & 0.6 & -0.09 & 0.5 \\
\hline
\end{tabular}

ms: millisecond, $\mu \mathrm{V}$ : microvolt.

Table 5. Correlation of PERG parameters with diabetes duration and the HbAlc level.

\begin{tabular}{|c|c|c|c|c|}
\hline \multirow{2}{*}{ PERG parameters } & \multicolumn{2}{|c|}{ Correlation with diabetes duration } & \multicolumn{2}{|c|}{ Correlation with the HbA1c level } \\
\hline & $\mathbf{r}$ & P-value & $\mathbf{r}$ & P-value \\
\hline $\mathrm{N} 35 \mathrm{~ms}$ & -0.1 & 0.3 & 0.17 & 0.2 \\
\hline $\mathrm{P} 50 \mathrm{~ms}$ & -0.02 & 0.8 & 0.16 & 0.2 \\
\hline N95 ms & 0.03 & 0.9 & 0.04 & 0.7 \\
\hline $\mathrm{N} 35 \mu \mathrm{V}$ & 0.1 & 0.3 & -0.08 & 0.5 \\
\hline $\mathrm{N} 35-\mathrm{P} 50 \mu \mathrm{V}$ & 0.07 & 0.5 & 0.2 & 0.1 \\
\hline P50-N95 $\mu \mathrm{V}$ & 0.004 & 0.9 & 0.2 & 0.1 \\
\hline PERG Ratio (N95: P50) & -0.19 & 0.1 & -0.1 & 0.3 \\
\hline
\end{tabular}

ms: millisecond, $\mu \mathrm{V}$ : microvolt.

\section{Discussion}

The VEP indicates the function of the entire visual pathway from the retina to the visual cortex and primarily reflects the central retinal projection to the occipital poles. P100 is usually a prominent peak that shows relatively little variation between subjects, minimal within-subject interocular difference, and minimal variation with repeated measurements over time $[13,16]$.

We have found in the present study that the mean (P100, $\mathrm{N} 135$ ) peak times in both (15' and 60') check sizes were statistically significantly prolonged in patients with type 2 DM without DR when compared to the control group. There were no statistically significant differences in mean N75 peak times of PRVEP between control and patients groups. These results are comparable to the findings of Gupta et al [17] who recorded PRVEP in 100 subjects (50 patients with type 2 diabetes without DR and 50 controls), mean age of diabetics was $51.6 \pm 9.48$ years and that of controls was $51.24 \pm 9.55$ years, he found a statistically significant increase in mean $\mathrm{P} 100$ latency $(\mathrm{P}<0.0001)$ as compared to the controls, mean N75 and N145 latencies showed an increase in diabetics but without statistical significance. These findings are also consistent with many previous similar studies including that by Heravian et al [18], Khatoon et al [19], Daniel et al [20], Gowri V [21], and Kothari et al [22], in their studies also reported a significant prolongation when they compared the mean P100 latency in diabetics without DR and controls. The difference between all studies and ours was the mean peak time values, variability could be explained by many factors like inclusion or exclusion criteria, sample sizes, and differences in recording conditions.

The mean peak times of (N75, P100, N135) were prolonged in type 2 diabetic patients with mNPDR in 
PRVEP15' but with no statistical significance. In the study by Lanting et al [23] who investigated P100 in 42 diabetic patients, they found no correlation between diabetic retinopathy and P100 latency. However, Daniel et al [20], and Kothari et al [22] found that P100 latencies were significantly prolonged in type 2 diabetic patients with DR when compared with that of control and the patients without DR. This difference between the studies could be for variability of sample size, and severty of retinopathy, or the presence of a significant proportion of patients with a primarily microvascular or microangiopathic phenotype, in which the role of neurodegeneration remains to be elucidated as explained by EUROCONDOR study [24].

The (N75, N75-P100, P100-N135) amplitudes of PRVEP $\left(60^{\prime}, 15^{\prime}\right)$ were decreased in diabetics especially group with mNPDR but with no statistical significance. When compared to mean P100 peak time prolongation in diabetics, reduction in its amplitude has been a less consistent finding in the previous studies $[18,19,20$, ]. There are great inter individual variations in the amplitudes of VEP compared with the peak time of responses. The VEP amplitude can be modified by attention, cranial shape, distribution of sulci of brain and size of the brain [19].

The PERG enables a more meaningful evaluation of a VEP, to exclude a macular cause of VEP abnormality and to provide an additional assessment of retinal ganglion cell involvement. The transient PERG has two major components of diagnostic value: P50 and N95. Both components reflect macular retinal ganglion cell function, but there is an additional more distal retinal contribution to the P50 component [16]. For PERG parameters we have found that diabetics had lower amplitudes but with no statiscal significance. The previous work by Jenkins et al [25] also showed no significant differences between the mean values of the diabetic patients and that of a normal age matched group in PERG. However, the study by Mermeklieva [26] demonstrated that PERG results were affected significantly in DM patients including the group without DR; abnormalities were more severe in patients with advanced DR.

There were some conflicting reports regarding correlation between diabetes duration or the $\mathrm{HbA1C}$ level and wave latencies or amplitudes of PRVEP. However, no statistically significant correlation was obtained between diabetes duration or the level of HbA1c and delay of peak times or reduce amplitudes in (PERG, PRVEP) with the exception for (N75-P100) amplitude in PRVEP15'.

The latency depends on an intact, myelinated nerve as myelin and saltatory conduction are essential for fast action potential propagation in normal subjects. In contrast, the amplitude of the waveform depends primarily on number of axons functioning within the nerve. Slowing of conduction velocity or prolongation of latency usually implies demyelinating injury, while loss of amplitude usually correlates with axonal loss dysfunction [21].

In an attempt to search for the pathogenetic mechanisms underlying the VEP abnormalities in diabetics, the studies in the past have concluded that responses observed in diabetics could be the expression of structural damage at the level of myelinated optic nerve fibers or retinal ganglion cell damage before the development of overt retinopathy.

Hyperglycemic milieu in diabetics results in the shunting of excess glucose into the polyol pathway and is converted to sorbitol and fructose. Sorbitol and fructose tend to accumulate within the nerve owing to the relative impermeability of the nerve cell membrane for the same. Osmotically active sorbitol and fructose increase the water content in the nerves. An associated depletion of nerve myoinositol also has been suggested. The above changes decrease the activity of $\mathrm{Na}+\mathrm{K}+\mathrm{ATP}$-ase (sodium potassium ATP-ase) thought to be located primarily in the nodal and paranodal regions of large myelinated nerve fibers resulting in increased intra-axonal $\mathrm{Na}+$ concentration, reduced nodal $\mathrm{Na}+$ permeability causing diminished conduction velocity.

Ischemic neuronal and other retinal structural damage caused by microvascular abnormalities in diabetes has also been implicated. Animal models of diabetic neuropathy have demonstrated that the neuropathy is accompanied by reduced endoneurial blood flow, increased endoneurial vascular resistance, and reduced oxygen tension. Ischemia may result in nerve fiber loss in peripheral nerves. It has been suggested that optic nerve fibers may also undergo similar ischemic changes in diabetes [17].

Neuropoietic cytokines including interleukin-1 (IL-1), IL-6, leukaemia inhibitory factor (LIF), ciliary neuro-trophic factor (CNTF), tumour necrosis factor alpha (TNF alpha), and transforming growth factor-beta (TGF-beta), exhibit pleiotropic effects on the homeostasis of the glia and on the neurons in the central, peripheral, and the autonomic nervous systems. These cytokines are produced locally by the resident and infiltrating macrophages, lymphocytes, mast cells, fibroblasts, and sensory neurons. The accumulation of these mediators delays the conduction in the visual pathway [21] and can culminate in the activation of caspases, exacerbating cell death in the retina [27].

In diabetes mellitus, damage occurs to ganglion cell layer which can be due to extracellular glutamate accumulation, leading to functional and anatomical changes, which rise even before the vascular damage. Oxidative stress, besides micro vascular abnormalities and consequences of glucose metabolism, play a great role in the pathological progress of diabetic retinopathy. That might be due to either an increase in free radical and oxidant production or reduced activity of anti-oxidative mechanisms [19].

\section{Conclusion}

We have found electrophysiological (PRVEP, PERG) alterations in diabetic patients when compared with those of the healthy. Therefore, these tests are sensitive and useful investigations for the early identification of visual dysfunctions before the development of retinopathy in type 2 diabetics. The differences in mean peak times (P100, N135) were statistically significant in PRVEP and thus it is more sensitive than PERG to detect these alterations. The 
electrophysiological evidence of visual dysfunctions in diabetics may indicate either dysfunction in the retinocortical conduction pathways and/or retinal damage.

This study suggests at least an annual PRVEP evaluation of diabetics without DR for a more complete and early assessment of the neurological involvement to advise them for an early and proper management of the disease. Future longitudinal studies should focus on VEP evaluation in the same diabetic patients in relation with diabetes duration and glycemic status, and detect the time that elapses between the appearance of the first detectable pathologic electrophysiologic changes and the first ophthalmoscopic detectable retinal changes.

\section{Conflicts of Interest}

All the authors do not have any possible conflicts of interest.

\section{References}

[1] Vanajarani, A. C. Evaluation of Retinal Ganglion Cell Activity by Pattern Visual Evoked Potential in Type2 Diabetic Patients. Chengalpattu Medical College, The Tamilnadu Dr. M. G. R. Medical University 2017.

[2] Aparna, A. Role of VEPs in Early Diagnosis of Central Neuropathy in Type 2 Diabetes Mellitus. Indian Journal of Public Health Research \& Development, October-December 2016; 7: 160-165.

[3] International Diabetes Federation. IDF Diabetes Atlas, 9th Edition. Brussels 2019.

[4] Umashankar; Gunasundarib, R. "A Review on Electrophysiology based Detection of Diabetic Retinopathy". Procedia Computer Science 2015; 48: 630-637.

[5] Yau, J. W; Rogers, S. L; Kawasaki, R; Lamoureux, E. L; Kowalski, J. W; Bek, T; Chen, Sh. J; Dekker, J. M; Fletcher, A; Grauslund, J; Haffner, S; Hamman, R. F; Ikram, M. K; Kayama, T; Klein, B. E; Klein, R; Krishnaiah, S; Mayurasakorn, K; O'Hare, J. P; Orchard, T. J; Porta, M; Rema M; Roy, M. S; Sharma, T; Shaw, J; Taylor, H; Tielsch, J. M; Varma, R; Wang, J. J; Wang, N. West, Sh; Xu, L; Yasuda, M; Zhang, X; Mitchell, P; Wong, T. Y. Meta-Analysis for Eye Disease (META-EYE) Study Group. Global prevalence and major risk factors of diabetic retinopathy. Diabetes Care 2012; 35: $556-564$.

[6] Abcouwer, S. F; Gardner, T. W. "Diabetic retinopathy: loss of neuroretinal adaptation to the diabetic metabolic environment" Ann N Y Acad Sci 2014; 1311: 174-190.

[7] Pescosolido, N; Barbato, A; Stefanucci, A; Buomprisco, G. "Role of Electrophysiology in the Early Diagnosis and Follow-Up of Diabetic Retinopathy". Journal of Diabetes Research 2015; Article ID 319692, 8 pages.

[8] Deák, K; Fejes, I; Janáky, M; Várkonyi, T; Benedek, G; Braunitzer, G. Further Evidence for the Utility of Electrophysiological Methods for the Detection of Subclinical Stage Retinal and Optic Nerve Involvement in Diabetes. Medical Principles and Practice, Vol. 25, 2016, 282-285.
[9] Simó, R; Hernández, C; Porta, M; Bandello, F; Grauslund, J; Harding, S. P; Aldington, S. J; Egan, C; Frydkjaer-Olsen, U; García-Arumí, J; Gibson, J; Lang, G. E; Lattanzio, R; Massin, P; Midena, E; Ponsati, B; Ribeiro, L; Scanlon, P; Lobo, C; Costa, M. Â; Cunha-Vaz, J. Effects of Topically Administered Neuroprotective Drugs in Early Stages of Diabetic Retinopathy: Results of the EUROCONDOR Clinical Trial. Diabetes 2019; 68: 457-463.

[10] Barber, A. J; Baccouche, B. "Neurodegeneration in diabetic retinopathy: Potential for novel therapies". Vision research 2017; 139: 82-92.

[11] Sohn, E. H; van Dijk, H. W; Jiao, C; Kok, P. H; Jeong, W; Demirkaya, N; Garmager, A; Wit, F; Kucukevcilioglu, M; van Velthoven, M. E; DeVries, J. H; Mullins, R. F; Kuehn, M. H; Schlingemann, R. O; Sonka, M; Verbraak, F. D; Abràmoff, M D. Retinal neurodegeneration may precede microvascular changes characteristic of diabetic retinopathy in diabetes mellitus. PNAS 2016, 2655-2664.

[12] American Academy of Ophthalmology. Retina and Vitreous, Basic and Clinical Science Courses Series (BCSC), San Francisco 2018-2019; 12: 303.

[13] Odom, J. V; Bach, M; Brigell, M; Holder, G. E; McCulloch, D. L; Meigen, T; Mizota, A; Tormene, A. P. "ISCEV standard for clinical visual evoked potentials: (2016 update)". Doc Ophthalmol 2016; 133: 1-9.

[14] Bach, M; Brigell, M. G; Hawlina, M; Holder, G. E; Johnson, M. A; McCulloch, D. L; Meigen, T; Viswanathan, S. "ISCEV standard for clinical pattern electroretinography (PERG): 2012 update". Doc Ophthalmol 2013; 126: 1-7.

[15] Instructions for Use and Maintenance-SISTEMA RETIMAX. retimographOPHT-06_User Manual \& Service Maual.

[16] Robson, A. G; Nilsson, J; Li, Sh; Jalali, S; Fulton, A. B; Tormene, A. P; Holder, G. E; Brodie, S. E. "ISCEV guide to visual electrodiagnostic procedures". Doc Ophthalmol 2018; 136: 1-26.

[17] Gupta, S; Khan, T; Gupta, G; Agrawal, B. K; Khan, Z. Electrophysiological evaluation in patients with type 2 diabetes mellitus by pattern reversal visual evoked potentials. Natl J Physiol Pharm Pharmacol 2017; 7.

[18] Heravian, J; Ehyaei, A; Shoeibi, N; Azimi, A; OstadiMoghaddam, H; Yekta, A; Khoshsima, M. J; Esmaily, H. Pattern Visual Evoked Potentials in Patients with Type II Diabetes Mellitus. J Ophthalmic Vis Res 2012; 7: 225-230.

[19] Khatoon, F; Bahmed, F; Katoon. N. Visual evoked potential as an early marker of diabetic retinopathy. Indian Journal of Clinical Anatomy and Physiology 2016; 3: 200-204.

[20] Daniel, R; Ayyavoo, S; Dass, B. Study of visual evoked potentials in patients with type 2 diabetes mellitus and diabetic retinopathy. Natl J Physiol Pharm Pharmacol 2017; 7: 159164.

[21] Gowri. Pattern Visual Evoked Potentials as a tool to assess the prognosis in Type 2 Diabetes Mellitus subjects attending a tertiary care hospital. University Journal of Pre and Para Clinical Sciences 2017; 3: 2455-2879.

[22] Kothari, R; Bokariya, P; Singh, S; Hemavaishnave, T. S. Evaluation of The Role Of Visual Evoked Potentials In Detecting Visual Impairment In Type II Diabetes Mellitus. Delhi Journal of Ophthalmology 2018; 28: 29-35. 
[23] Lanting, P; Strijers, R. L; Bos, J. E; Faes, T. J; Heimans, J. J. The cause of increased pupillary light reflex latencies in diabetic patients: the relationship between pupillary light reflex and visual evoked potential latencies. Electroencephalography and Clinical Neurophysiology 1991; 78: $111-115$.

[24] Santos, A. R; Ribeiro, L; Bandello, F; Lattanzio, R; Egan, C; Frydkjaer-Olsen, U; García-Arumí, J; Gibson, J; Grauslund, J; Harding, S. P; Lang, G. E; Massin, P; Midena, E; Scanlon, P; Aldington, S. J; Simão, S; Schwartz, Ch; Ponsati, B; Porta, M; Costa, M. Â; Hernández, C; Cunha-Vaz, J; Simó, R. Functional and Structural Findings of Neurodegeneration in Early Stages of Diabetic Retinopathy. Cross-sectional analyses of Baseline Data of the EUROCONDOR project. Diabetes 2017.

[25] Jenkins, T. C. A; Cartwright, J. P. The electroretinogram in minimal diabetic retinopathy. British Journal of Ophthalmolology 1990; 74: 681-684.

[26] Mermeklieva, E. A. Pattern electroretinography and retinal changes in patients with diabetes mellitus type2. Neurophysiologie Clinique/Clinical Neurophysiology 2019; 49: 209-215.

[27] Lechner, J; O'Leary, O. E; Stitt, A. W. "The pathology associated with diabetic retinopathy". Vision Research 2017; 139: 7-14. 